\title{
General Closed-Form Solutions to the Dynamic Optimization Problem in Incomplete Markets
}

\author{
Moawia Alghalith \\ Economics Department, University of the West Indies (UWI), St. Augustine, Trinidad and Tobago \\ E-mail:malghalith@gmail.com \\ Received January 5, 2011; revised February 23, 2011; accepted February 25, 2011
}

\begin{abstract}
In this paper, we provide general closed-form solutions to the incomplete-market random-coefficient dynamic optimization problem without the restrictive assumption of exponential or HARA utility function. Moreover, we explicitly express the optimal portfolio as a function of the optimal consumption and show the impact of optimal consumption on the optimal portfolio.
\end{abstract}

Keywords: Stochastic, Incomplete Markets, Investment

\section{Introduction}

Dynamic optimization has been used extensively in the economic and financial literature. Examples include incomplete markets, stochastic volatility and random coefficients models. The contemporary literature usually adopts random coefficient models (the parameters of the model are dependent on a random external economic factor) or non-tradable assets models. Examples include Bayraktar and Young [1], Bayraktar and Ludkovsky [2], Alghalith [3], Focardi and Fabozzi [4], and Fleming [5].

In order to derive explicit solution to the optimization problem, the previous studies relied exclusively on exponential or HARA utility functions. This assumption is restrictive and sometimes unrealistic, since other common and more appropriate functional forms exist.

In this paper, we relax the exponential or HARA utility assumption. In doing so, we derive general closed form solutions to the random-coefficient incompletemarket dynamic optimization problem without imposing restrictions on the functional form of utility. Furthermore, we explicitly derive a functional relationship between the optimal portfolio and optimal consumption and show the impact of consumption on the optimal portfolio.

In Section 2, we present the theoretical model and the results. In Section 3, we draw conclusions.

\section{The Model}

We consider a standard investment-consumption model, which includes a risky asset, a risk-free asset and a random external economic factor (see, for example, Fleming [5] for background information on the investment-consumption model). This implies a two-dimensional standard Brownian motion $\left\{\left(W_{s}^{1}, W_{s}^{2}\right), \mathcal{F}_{s}\right\}_{t \leq s \leq T}$ on the probability space $\left(\Omega, \mathcal{F}_{s}, P\right)$, where $\left\{\mathcal{F}_{s}\right\}_{t \leq s \leq T}$, is the augmentation of filtration. The risk-free asset price process is $S_{0}=e^{\int_{t}^{T} r\left(Y_{s}\right) \mathrm{d} s}$, where $r\left(Y_{s}\right) \in C_{b}^{2}(R)$ is the rate of return and $Y_{s}$ is the economic factor.

The risky asset price process is given by

$$
\mathrm{d} S_{s}=S_{t}\left\{\mu\left(Y_{s}\right) \mathrm{d} t+\sigma\left(Y_{s}\right) \mathrm{d} W_{s}^{1}\right\},
$$

where $\mu\left(Y_{t}\right)$ and $\sigma\left(Y_{t}\right)$ are the rate of return and the volatility, respectively. The economic factor process is given by

$$
\mathrm{d} Y_{s}=b\left(Y_{s}\right) \mathrm{d} t+\rho \mathrm{d} W_{s}^{1}+\sqrt{1-\rho^{2}} \mathrm{~d} W_{s}^{(2)}, Y_{t}=y,
$$

where $|\rho|<1$ is the correlation factor between the two Brownian motions and $b\left(Y_{s}\right) \in C^{1}(R)$ with bounded derivative.

The wealth process is given by

$$
b\left(Y_{s}\right) \in C X_{T}^{\pi, c}=x+\int_{t}^{T}\left\{r\left(Y_{s}\right) X_{s}^{\pi, c}+\left(\mu\left(Y_{s}\right)-r\left(Y_{s}\right) \pi_{s}\right)-c_{s}\right\} \mathrm{d} s+\int_{t}^{T} \pi_{s} \sigma\left(Y_{s}\right) \mathrm{d} W_{s}^{1},
$$


where $x$ is the initial wealth, $\left\{\pi_{t}, \mathcal{F}_{s}\right\}_{t \leq s \leq T}$ is the portfolio process and $\left\{c_{t}, \mathcal{F}_{s}\right\}_{t \leq s \leq T}$ is the consumption process, with $\int_{t}^{T} \pi_{s}^{2} \mathrm{~d} s<\infty, \int_{t}^{T} c_{s} \mathrm{~d} s<\infty$ and $c \geq 0$. The trading strategy $\left(\pi_{s}, c_{s}\right) \in \mathcal{A}(x, y)$ is admissible (that is, $\left.X_{s}^{\pi, c} \geq 0\right)$.

The investor's objective is to maximize the expected utility of wealth and consumption

$$
V(t, x, y)=\operatorname{Sup}_{\pi_{t}, c_{t}} E\left[u_{1}\left(X_{T}^{\pi, c}\right)+\int_{t}^{T} u_{2}\left(c_{s}\right) \mathrm{d} s \mid \mathcal{F}_{t}\right],
$$

where $V($.$) is the value function, u($.$) is a differenti-$ able, bounded and strictly concave utility function.

The value function satisfies the Hamiltonian-JacobiBellman PDE

$$
V_{t}+r(y) x V_{x}+g(y) V_{y}+\frac{1}{2} V_{y y}+\operatorname{Sup}_{\pi_{t}, c}\left\{\frac{1}{2} \pi_{t}^{2} \sigma^{2}(y) V_{x x}+\left[\pi_{t} \sigma(y) \theta(y)-c_{t}\right] V_{x}+\rho \sigma(y) \pi_{t} V_{x y}+u_{2}\left(c_{t}\right)\right\}=0,
$$

$$
V(T, x, y)=u(x),
$$

where $\theta\left(Y_{t}\right) \equiv \sigma^{-1}\left(Y_{t}\right)\left(\mu\left(Y_{t}\right)-r\left(Y_{t}\right)\right)$. Differentiating the above equation with respect $\pi_{t}$ and $c_{t}$, respectively and rearranging, we obtain the optimal solutions

$$
\begin{aligned}
& \pi_{t}^{*}=-\sigma^{-1}(y) \frac{\theta(y) V_{x}+\rho V_{x y}}{V_{x x}}, \\
& \qquad u_{2}^{\prime}\left(c_{t}^{*}\right)=V_{x} . \\
& \min _{c_{t}}\left\{R(x)=\left(u_{2}\left(c_{t}\right)-\left[u_{2}(a)\right.\right.\right. \\
& \text { solution yields } \\
& R^{\prime}\left(\hat{c}_{t}\right)=u_{2}^{\prime}\left(\hat{c}_{t}\right)-u_{2}^{\prime}(a)-u_{2}^{\prime \prime}(a)\left(\hat{c}_{t}-a\right)=0,
\end{aligned}
$$$$
\min _{c_{t}}\left\{R(x)=\left(u_{2}\left(c_{t}\right)-\left[u_{2}(a)+u_{2}^{\prime}(a)\left(c_{t}-a\right)+\frac{1}{2} u_{2}^{\prime \prime}(a)\left(c_{t}-a\right)^{2}\right]\right)\right\} .
$$

Lemma. We can obtain an exact fixed-coeffiecient Taylor expansion of $u_{2}\left(c_{t}^{*}\right)$.

Proof. Consider the following Taylor expansion around $a$

$$
\begin{aligned}
u_{2}\left(c_{t}\right)= & u_{2}(a)+u_{2}^{\prime}(a)\left(c_{t}-a\right) \\
& +\frac{1}{2} u_{2}^{\prime \prime}(a)\left(c_{t}-a\right)^{2}+R\left(c_{t}\right),
\end{aligned}
$$

where $R\left(c_{t}\right)$ is the remainder. Our objective is to minimize $R\left(c_{t}\right)$
The solution yields

and thus

$$
u_{2}^{\prime}\left(\hat{c}_{t}\right)=u_{2}^{\prime}(a)+u_{2}^{\prime \prime}(a)\left(\hat{c}_{t}-a\right) .
$$

Now since $\hat{c}_{t}$ depends on the value of $a$, choose a specific value of $a=\bar{a}$ such that $\hat{c}_{t}=c_{t}^{*}$; hence

$$
u_{2}^{\prime}\left(c_{t}^{*}\right)=u_{2}^{\prime}(\bar{a})+u_{2}^{\prime \prime}(\bar{a})\left(c_{t}^{*}-\bar{a}\right) \text {. }
$$

The above equation can be rewritten as

$$
u_{2}^{\prime}\left(c_{t}^{*}\right)=b_{1}+b_{2} c_{t}^{*},
$$

where $b_{i}$ is a constant. Using the same procedure we obtain the following exact expansion of $V_{x}($.

$$
\begin{aligned}
V_{x}(.) & =V_{x}(\alpha)+V_{x x}(\alpha)\left(x-\alpha_{1}\right)+V_{x y}(\alpha)\left(y-\alpha_{2}\right) \\
& \equiv b_{3}+b_{4} x+b_{5} y .
\end{aligned}
$$

Since $u_{2}^{\prime}\left(c_{t}^{*}\right)=V_{x}($.$) , we obtain$

$$
c_{t}^{*}=\left(b_{6}+b_{4} x+b_{5} y\right) / b_{2} .
$$

Substituting (2) and (3) into (1) yields

$$
\pi_{t}^{*}=-\sigma^{-1}(y) \frac{\theta(y)\left(b_{1}+b_{2} c_{t}^{*}\right)+b_{5} \rho}{b_{4}} .
$$

This is a general explicit formula that holds for any utility function. Moreover, this formula allows us to determine the impact of consumption on the portfolio. To show this

$$
\frac{\partial \pi_{t}^{*}}{\partial c_{t}^{*}}=-\sigma^{-1}(y) \theta(y) \frac{b_{2}}{b_{4}} \equiv-\sigma^{-1}(y) \theta(y) \frac{u_{2}^{\prime \prime}(\bar{a})}{V_{x x}(\alpha)}<0
$$

by the concavity of $u$.

This approach is empirically very convenient since the parameters in (4) $b_{1}, b_{2}, b_{4}$ and $b_{5}$ can be easily estimated using a nonlinear regression based on historical data.

\section{Conclusion}

This approach is superior to the existing approaches in several ways. First, we can obtain closed-form solutions without the assumption of exponential or HARA utility function. Secondly, we can easily obtain comparative statics results. For example, there is a trade-off between consumption and investment. Moreover, our approach can be easily utilized by future empirical studies.

\section{References}

[1] E. Bayraktar and V. Young, “Optimal Investment Strate- 
gy to Minimize Occupation Time," Annals of Operations Research, Vol. 176, No. 1, 2010, pp. 389-408. doi:10.1007/s10479-008-0467-2

[2] E. Bayraktar and M. Ludkovski, "Inventory Management with Partially Observed Nonstationary Demand," Annals of Operations Research, Vol. 176, No. 1, 2010, pp. 7-39. doi:10.1007/s10479-009-0513-8

[3] M. Alghalith, "A New Stochastic Factor Model: General Explicit Solutions,” Applied Mathematics Letters, Vol. 22,
No. 12, 2009, pp. 1852-1854. doi:10.1016/j.aml.2009.07.011

[4] F. Focardi and F. Fabozzi, "The Mathematics of Financial Modeling and Investment Management,” Wiley E-Series, 2004.

[5] W. Fleming, "Some Optimal Investment, Production and Consumption Models," Contemporary Mathematics, Vol. 351, 2004, pp 115-124. 